\title{
Simple Statistical Model for Complex Probabilistic Climate Projections: Overheating Risk and Extreme Events
}

\author{
Sandhya Patidar ${ }^{1, *}$, David Jenkins ${ }^{2}$, Phil Banfill ${ }^{2}$, Gavin Gibson ${ }^{1}$ \\ ${ }^{1}$ Maxwell Institute for Mathematical Sciences , School of Mathematical and Computer Sciences, Heriot- \\ Watt University, Edinburgh, UK \\ ${ }^{2}$ Urban Energy Research Group, School of Built Environment (address as above) \\ * Corresponding author. Tel: +44 1314514365, E-mail: S.Patidar@hw.ac.uk
}

\begin{abstract}
Climate change could substantially impact the performance of the buildings in providing thermal comfort to occupants. Recently launched UK climate projections (UKCP09), clearly indicate that all areas of the UK will get warmer in future with the possibility of more frequent and severe extreme events, such as heat waves. This study, as part of the Low Carbon Futures (LCF) Project, explores the consequent risk of overheating and the vulnerability of a building to extreme events. A simple statistical model proposed by the LCF project elsewhere has been employed to emulate the outputs of the dynamic building simulator (ESP-r) which cannot feasibly be used itself with thousands of available probabilistic climate database. Impact of climate change on the daily external and internal temperature profiles has been illustrated by means of 3D plots over the entire overheating period (May - October) and over 3000 equally probable future climates. Frequency of extreme heat events in changing climate and its impact on overheating issues for a virtual case study domestic house has been analyzed. Results are presented relative to a baseline climate (1961-1990) for three future timelines (2030s, 2050s, and 2080s) and three emission scenarios (Low, Medium, and High).
\end{abstract}

Keywords: Probabilistic climate projections, Building and Adaptation, Overheating

\section{Introduction}

Experiments based on an advanced scientific methodology and general circulation models (GCM), commonly known as global climate models, reveal that there is an exponential increase in the global concentration of greenhouse gases [1]. According to a UK Climate Projections (UKCP09) [2] briefing report, central England's temperature has already increased by $1{ }^{\circ} \mathrm{C}$ since the 1970 s and this increase is most likely due to anthropogenic emission of greenhouse gases [3]. This excessive addition of the greenhouse gasses in the atmosphere is warming up the Earth's surface causing climate change and thus altering the weather pattern. Climate change could hasten species extinction, cause coastal flooding, and lead to more frequent and severe storms and extreme temperature events such as heat waves. Such extreme temperature events could cause catastrophic losses to both human and natural systems and thereby have dramatic ecological, economic and sociological impact [3-8].

For the UK, projections for future changes to climate are provided by the UK Climate Projections (UKCP09) and are available in probabilistic format to address the uncertainty associated with future climate change. To include a measure for the uncertainty, these climate projections are generated by multiple simulations of global climate models (in particular HadCM3 [9]) combined with a new methodology designed by the Met Office. The projections also include the results of other IPCC [1] climate models, and are constrained by observations of past climate. Thus to describe just any single future climate scenario a range (in fact thousands) of possible climates are available where each climate has a certain probability of occurring.

Extreme events, by definition, are in the tails of such probability distributions. Interestingly, events in the tail of the distribution are the ones that change most in frequency of occurrence as the distribution shifts due to global warming [10-11]. The work presented in this paper will 
focus on the potential impact of future climate changes on the frequency of the extreme temperature regimes (heat wave) and how this could influence the overheating issues in the UK's domestic house sectors.

The suite of probabilistic climates projection available from UKCP09 for a London location at three future timelines, namely 2030's, 2050s, and 2080s, including a baseline [1960-1990] has been carefully analyzed to quantify the future change in the frequency of extreme heat events. The impact of extreme heat events on indoor comfort temperatures of a domestic building case study has been assessed by means of a simple statistical model proposed by the Low Carbon Futures (LCF) project [12] elsewhere [13-14]. This simple statistical tool is mainly based on multiple regression techniques and could efficiently emulate the outputs of traditional dynamics building simulation software ESP-r [15], which cannot be practically used with thousands of potential climates available for each future scenario.

This project is sponsored by the Adaptation and Resilience in a Changing Climate (ARCC) Programme [16], looking at possible methods, i.e. adaptations, for coping with a future climate.

\section{Methodology}

To analyze complex probabilistic climate information and to generate corresponding indoor temperature profiles for a case study dwelling, an elegant regression tool has been developed by the LCF project which is described in detail elsewhere [14]. The simple regression tool which is based on data reduction methods such as Principal Component Analysis (PCA) and multiple regressions is validated at an hourly scale to provide a close match (within a range of $1{ }^{\circ} \mathrm{C}$ ) for the outputs of dynamic building simulation software (ESP-r). More details on the development procedure of the regression tool are found elsewhere [13].

This section should present a brief description of probabilistic climatic information available from UKCP09, profiles of the domestic building case study simulated with ESP-r, and a short note on the regression tool which has been employed to emulate outputs of ESP-r for quantifying extreme heat events and its impact on overheating issues in the case-study building. A short review on the possible definitions of the heat wave is also included.

\subsection{Probabilistic Climate Projections and Regression Tool}

The UKCP09 provides climate change information focused on the UK. The projections are presented for seven 30 years time periods ("2020s" denoting as 2010 - 2039, “2030s" denoting 2020 - 2049, ... , up to "2080s" denoting 2070 - 2099), and at three different future greenhouse gas emissions scenarios represented as "High", "Medium" and "Low". These projections are based on change relative to a baseline time period (1961-1990) and, by means of a Weather Generator (WG) [17] tool, are available at an hourly temporal resolution with a $5 \times 5 \mathrm{~km}^{2}$ spatial resolution for any user defined UK location.

For the work presented in this article, climatic information has been downloaded from UKCP09's WG tool for a London location corresponding to all three greenhouse gas emissions scenarios and at three future time periods, namely 2030s, 2050s and 2080s, including baseline. Notably for any user specified future scenario, the WG tool could provide at least 3000 equally probable hourly climate files (each climate file represents a prototype climate year for that scenario) in the form of 100 statistically equivalent time series, where each of the time series is equally probable and of 30 years in length. For each of the future scenarios under investigation a representative sample of 100 climate years is formed by means 
of a random sampling algorithm, which selects one year randomly from each of the 100 time series.

To formulate the regression tool, a 3-bedroom cavity-wall house with a total floor area of 144 $\mathrm{m}^{2}$ has been simulated with ESP-r for one randomly chosen climate file from the 100 available climate years (additional information on the virtual case study building can be found elsewhere [18]). The regression tool is based on the concept of underpinning the existing relationship between available climate variants and the indoor temperatures, where each climate variant first need to be carefully analyzed and simplified by means of PCA. The model has been formulated to capture indoor temperature profiles during the period of May to October only. To allow time-based sensitivity analysis, the idea of segmented modelling has been applied and data fitted in parts, namely a) May - June; b) July-August; and c) September - October, to cover the entire period (May-October) [13].

A regression tool which could be formulated from the one climate file had been validated across all the available 100 climates files and corresponding to all different future scenarios as described above. Moreover, the proposed statistical regression tool has been demonstrated to efficiently estimate the outputs of ESP-r at an hourly scale within a single degree centigrade for up to $90 \%$ of the entire data set [13-14]. Thus, it is reasonable to consider an application of the proposed simple and efficient regression tool for quantifying frequency of heat waves and analyzing overheating issues in that context.

\subsection{Heat waves and Overheating}

Research conducted by Met Office clearly shows that even in the UK heat waves could be lethal, when hotter summers are predicted in foreseeable future [19]. There is no universal definition of a heat wave, however a heat wave could be considered as a prolonged period of excessively hot weather accompanied by high humidity, where temperatures are outside the normal climate pattern for that period [20]. For the UK, the Met Office defines summer heat wave duration as "the sum of days with daily maximum temperature more than $3^{\circ} \mathrm{C}$ above 1961-90 daily normal for $\geq 5$ consecutive days (May-October)" [21].

As stated previously, extreme events formulate tails of the probability distribution and therefore for the present study of heat waves and overheating analysis, it is essential to consider all 3000 climates corresponding to each available future scenario. An initial analysis of 3000 baseline climate files identified $15.6{ }^{\circ} \mathrm{C}$ as the daily normal temperature for 1961 1990. For each climate file daily normal temperature has been averaged over the overheating period and then again averaged over all equally probable 3000 climate years.

Thus, summer heat wave duration could be defined as - "sum of days with daily maximum temperature more than $18.6^{\circ} \mathrm{C}$ for 5 or more days during May to October". In this context, to examine the impact of heat waves on indoor temperatures, two overheating criterion had been designed and compared with corresponding heat wave durations:

Overheating Criterion 1 - "Sum of the days with average of night time (11pm - 7am) bedroom temperature more than $24{ }^{\circ} \mathrm{C}$ for 5 or more days during May to October".

Overheating Criterion 2 - "Sum of the days with average of night time (11pm - 7am) bedroom temperature more than $28^{\circ} \mathrm{C}$ for 5 or more days during May to October".

Notably, overheating Criteria 1 and 2 are designed by the author and are mainly motivated from the Met Office definition of summer heat wave in order to compare the influence of external climates on the indoor comfort temperatures. 


\section{Result}

Following the above definition of a heat wave and overheating criteria, some key results has been presented in this section for a London location.

\subsection{External and Internal Temperatures}

An analysis of external and internal temperatures are performed at an hourly scale and presented at daily scale corresponding to 3000 climate years (during summer period, May October) by means of 3D color coded plots in Figs. 1 and 2. Climatic files have been analyzed to measure change in daily maximum external temperature and corresponding average night time (11pm - 7am) bedroom temperatures in three future time periods: 2030s, 2050s and 2080s, in relation to a baseline period for the medium emission scenario.

\section{London - Daily Maximum External Temperature}
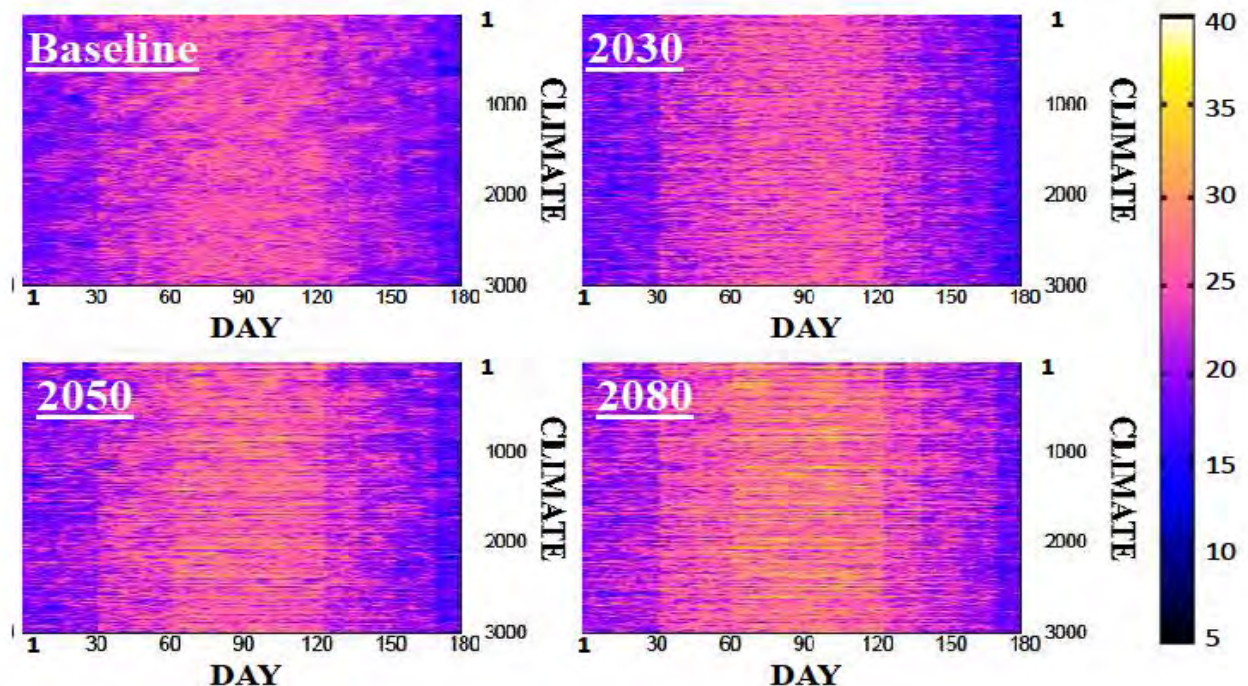

Fig. 1. Daily maximum of external temperature measured over 3000 climates for London - Medium Emission Scenario.

A visual inspection of Fig. 1 suggests that, for the baseline period daily maximum external temperature varies between $10-20{ }^{\circ} \mathrm{C}$. Notably, temperatures around $10{ }^{\circ} \mathrm{C}$ could be observed as a less probable event on a distribution curve of the entire dataset, however it can be easily confirmed from the $3 \mathrm{D}$ plots that $10{ }^{\circ} \mathrm{C}$ is the most probable temperature for the start and end of the period i.e. May and October. During the 2030s, a temperature range of $15-20{ }^{\circ} \mathrm{C}$ appears to predominate, whereas during the 2050s, maximum external temperature could reach $30{ }^{\circ} \mathrm{C}$, though less often and mainly during peak summer time (July - August). Events of maximum external temperature reaching $30{ }^{\circ} \mathrm{C}$ during peak summer period (July-August) could become more frequent in 2080s.

A simple statistical tool (as described in Section 2) has been used to reflect the changes in the averaged night time bedroom temperatures over the corresponding three future time periods: 2030s, 2050s and 2080s, in relation to the baseline period Fig. 2. Visual inspection of Fig. 2 suggests that for the baseline period the most probable average night time bedroom temperature ranges is $15-20{ }^{\circ} \mathrm{C}$ (May and October), $20-25{ }^{\circ} \mathrm{C}$ (June and September), and 25 - $27^{\circ} \mathrm{C}$ (July and August). However, for the 2030s the most probable temperature range appears to be $20-25{ }^{\circ} \mathrm{C}$, whereas $25-30{ }^{\circ} \mathrm{C}$ appear to be dominating during peak summer period (July - August). In the 2050s, the most probable temperature range still appears to be 
20 - $25{ }^{\circ} \mathrm{C}$ with more probabilities, in comparison to 2030s, shifted to the temperature range 25 - $30{ }^{\circ} \mathrm{C}$ during peak summer period (July - August). The impact of climate change could further raise the probability attached to temperature ranges $25-30{ }^{\circ} \mathrm{C}$ in 2080s with the peak summer period (July and August) mainly dominated by the temperatures above $30^{\circ} \mathrm{C}$.

\section{London - Average Bedroom Temperature during Night}
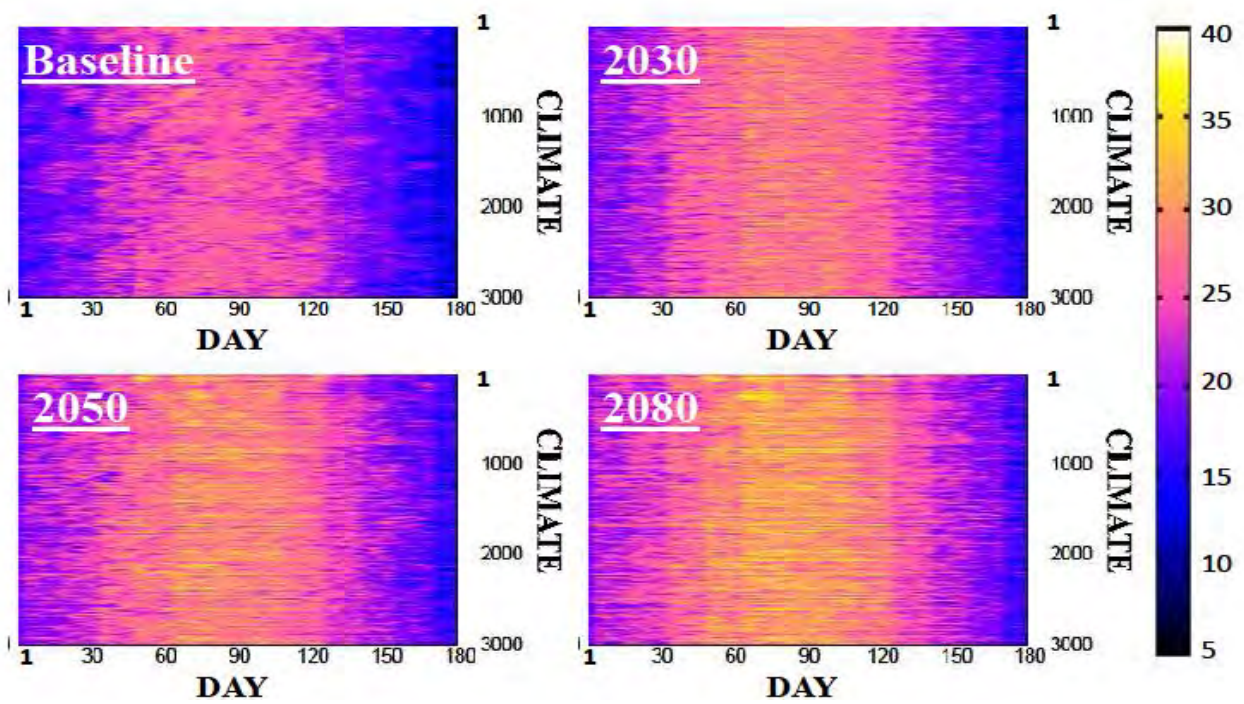

Fig. 2. Daily average of bedroom temperature during night (11pm-7am) measured over 3000 climates for London - Medium Emission Scenario.

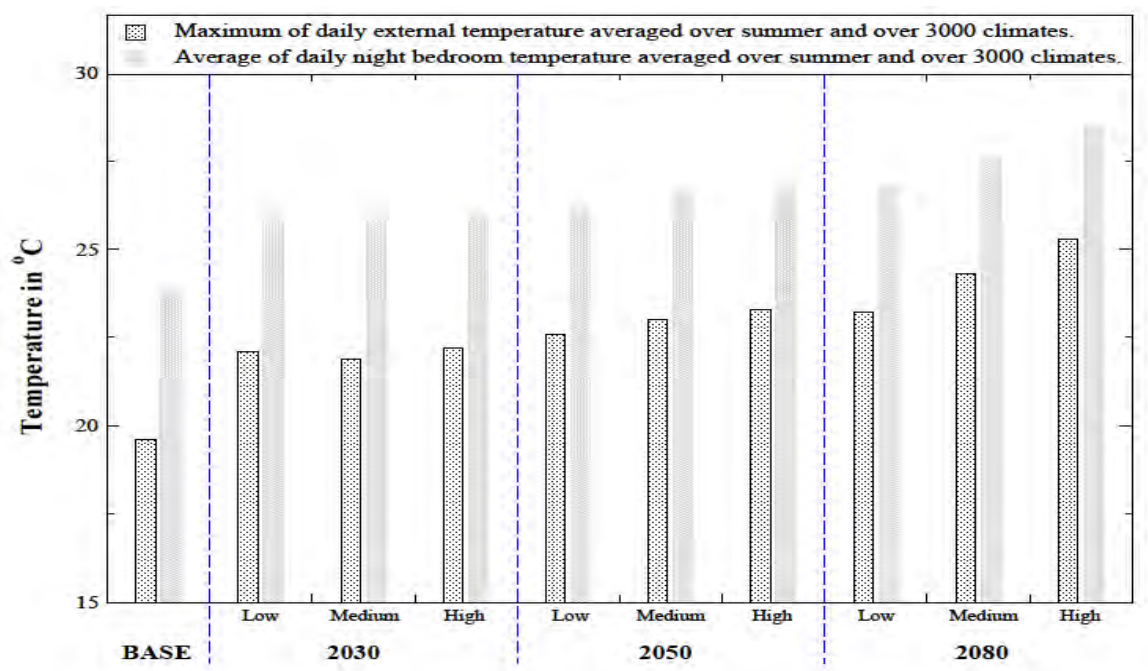

Fig. 3. Averaged daily maximum external temperature and average night bedroom temperature. Averaged over summer period and over 3000 climate years for three future time periods (2030s, 2050s, 2080s), three emission scenarios (Low, Medium, High) and baseline(1960-1990). Location: London

Finally, Fig. 3 shows the average maximum daily external temperature and corresponding average night bedroom temperature across three future time periods considered in the paper, alongside three carbon emission scenarios and the baseline period. For each scenario the average daily measurements for internal and external temperature parameters are firstly calculated over the May to October period and then over all 3000 climate years. The average of maximum daily external temperature in medium emission scenario rises to approximately $22{ }^{\circ} \mathrm{C}$ (2030s), $23{ }^{\circ} \mathrm{C}$ (2050s), and $24{ }^{\circ} \mathrm{C}$ (2080s) from $19{ }^{\circ} \mathrm{C}$ (baseline period). Average night 
bedroom temperature in medium emission scenario approximately rises to $26{ }^{\circ} \mathrm{C}$ (2030s), 27 ${ }^{\circ} \mathrm{C}$ (2050s), and $28{ }^{\circ} \mathrm{C}$ (2080s) from $24{ }^{\circ} \mathrm{C}$ (baseline period).

\subsection{Heat wave and Overheating Analysis}

This subsection presents a complete analysis of summer heat wave duration and its impact on consequent overheating duration defined in criterion 1 and 2. The result has been presented across three future time periods (2030s, 2050s, and 2080s), three emission scenarios (upper panel), and the baseline period.

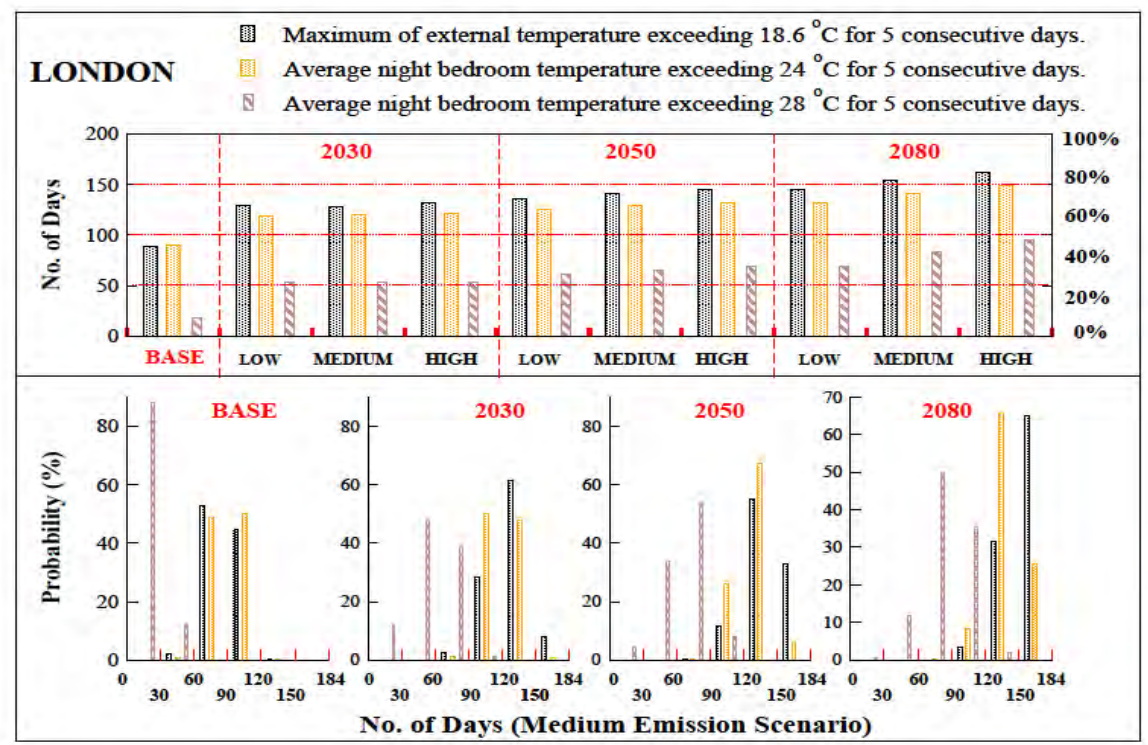

Fig. 4. Summer heat wave duration and overheating criterion 1 and 2 measured and compared. Dotted black bar: summer heat wave duration, gray (yellow online) dotted bars: overheating criterion 1, and gray (brown online) filled with slanted lines: overheating criterion 2.

In Fig. 4 the upper panel quantifies averaged (taken over 3000 climates) summer heat wave duration, overheating duration (defined in criterion 1 and 2) across all scenarios, in terms of total number of days during the identified period (May to October) along the left hand side of the $x$ - axis, and in \% of the May - October period along the right hand side of the $x$ - axis. Dot filled black bars measure the averaged summer heat wave duration, while gray (yellow online) dotted bars measure the average duration of overheating defined under criterion 1 and gray (brown online) bars filled with slanted lines represents the average duration of overheating defined under criterion 2. Heat-wave duration and overheating duration defined in criterion 1 show similar trends in different future scenarios and, in particular, for medium emission scenario appears to rise by approximately $20 \%$ (2030s), $30 \%$ (2050s), and 40\% (2080s) of total period in relation to baseline period. Overheating duration defined in criterion 2 increased by $15 \%$ (2030s), $20 \%$ (2050s), and $30 \%$ (2080s) of total period in relation to baseline period.

Fig. 4 lower panel displays variation in summer heat wave duration, overheating duration (defined in criterion 1 and 2) across medium scenario for baseline, 2030s, 2050s and 2080s, distributed along total number of days during the period (May to October) for London medium emission scenario. For a total of more than $90 \%$ central probability over 3000 climates, summer heat wave duration and overheating duration defined in criterion 1 could shift from 60 - 120 days in the baseline period to 90 - 120 days in 2030s, to 90 - 184 days in 2050s, and to 120 - 184 days in 2080s. Overheating duration defined in criterion 2 could shift 
from 30 - 60 days in baseline period to 30 - 90 days in 2030s, to 60 - 90 days in 2050s, and to 90 - 120 days in 2080s.

\section{Discussion and Conclusions}

This study presents an application of the simple statistical model which forms part of a methodology proposed by the Low Carbon Futures project to integrate future climate projections into building design and simulation. The statistical model presented in the form of a simple linear predictor has been used to describe the influence of climate change on the pattern of extreme heat events and its impact on the indoor comfort temperatures for a simple domestic building case study. Heat-wave duration and overheating duration defined under two distinct temperature based criteria in section 2.2 have been used as appropriate measures.

The results presented in Section 3.1 clearly illustrate the impact of climate change on the daily external and internal temperature profiles by means of 3D (color online) plots for given probabilistic climate projections. The average of maximum daily external temperature in the medium emission scenario appears to increases to $22{ }^{\circ} \mathrm{C}-24{ }^{\circ} \mathrm{C}$ (2030s to 2080s) from $19{ }^{\circ} \mathrm{C}$ (baseline period), whereas average night bedroom temperature could reach $26{ }^{\circ} \mathrm{C}-28{ }^{\circ} \mathrm{C}$ (2030s to 2080s) from $24{ }^{\circ} \mathrm{C}$ (baseline period).

In section 3.2 the summer heat-wave duration and overheating duration defined in criterion 1 appears to display a similar trend and up to a $20 \%-40 \%$ rise in total overheating period (2030s to 2080s) in relation to baseline period has been observed, whereas for the overheating duration defined in criterion 2 a 15\% - 30\% rise has been noticed. Outputs displayed in probabilistic format clearly show an increase in the total number of heat-wave duration days and overheating duration days defined in criteria 1 and 2.

Notably, the statistical model employed for overheating analyses has been demonstrated to replicate the outputs of ESP-r at an hourly scale within a single degree centigrade. The work presented in this paper could be used to develop methodologies for sustainable building design which could achieve a more acceptable level of thermal comfort in extreme climates. The effects of various adaptation techniques could be assessed to offset the predicted risk of overheating and in combating the influence of extreme events. Moreover, a range of possible climates, location and building variants could be investigated following the proposed methodology.

\section{References}

[1] IPCC, IPCC Fourth Assessment Report: Climate Change, 2007. Online at: http://www.ipcc.ch/publications and data/publications and data reports.htm

[2] J. Murphy et al., UK Climate Change Projections science report: Climate Change Projections., Technical report, Meteorological Office Hadley Centre, Exeter, UK, 2009.

[3] G. J. Jenkins, J. M. Murphy, D. M. H. Sexton, J. A. Lowe, P. Jones, and C. G. Kilsby, UK Climate Projections: Briefing report, Met Office Hadley Centre, Exeter, UK (2009). Online

at: http://ukclimateprojections.defra.gov.uk/images/stories/briefing_pdfs/UKCP09_Briefing. pdf

[4] D. R. Easterling, G. A. Meehl, C. Parmesan, S. A. Changnon, T. R. Karl, and L. O. Mearns, Science 289, 2000, pp. 2068 - 2074.

[5] L. S. Kalkstein, and J. S. Greene, Environ. Health Perspect. 105, 1997, pp. 84 - 93. 
[6] G. R. Walther, E. Post, P. Convey, A. Menzel, C. Parmesan, T. J. C. Beebee, J. M. Fromentin, O. Hoegh-Guldberg, and F. Bairlein, Nature 416, 2002, pp. 389 - 395.

[7] A. T. DeGaetano, Int. J. Biometeorol. 49, 2005, pp. 345 - 353.

[8] P. P. Marra, S. Griffing, C. Caffrey, A. M. Kilpatrick, R. McLean, C. Brand, E. Saito, A. P. Dupuis, L. Kramer, and R. Novak, Bioscience 54, 2004, pp. 393 - 402.

[9] Hadley centre coupled ocean atmosphere global climate model is known as HadCM3.

[10]2010 - How Warm Was This Summer? NASA Goddard Institute for Space Studies (GISS), 28 September 2010. See also How Warm Was Summer 2010?, Research News (30 Sep 2010) from NASA GISS. Online at: http://data.giss.nasa.gov/gistemp/

[11]J. Hansen, R. Ruedy, M. Sato, and K. Lo, Global Surface temperature Change, NASA Goddard Institute for Space Studies, New York, New York, USA. Online at: http://data.giss.nasa.gov/gistemp/paper/gistemp2010_draft0803.pdf

[12]LCF, Low Carbon Future Project, 2009. Online: http://www.ukciparcc.org.uk/index.php?option=com contenttask=viewid=589Itemid $=542$

[13]S. Patidar, D. P. Jenkins, G. J. Gibson, P. F. G. Banfill, Statistical techniques to emulate dynamic building simulations for overheating analyses in future probabilistic climates, Journal of Building Performance Simulation, 1-14, iFirst article, 2011.

[14]D. P. Jenkins, S. Patidar, G. J. Gibson, P. F. G. Banfill, Incorporating future probabilistic climate projections into dynamic building simulation, Energy and Buildings, in correspondence.

[15]J. Clarke, N. Kelly, and D. Tang, A review of ESP-rs exible Solution Approach and its Application to Prospective Technical Domain Developments V1, In: Advances in Building Energy Research. UK: Earthscan, 2007, chap. 11.

[16]ARCC, Adaptation and Resilience in a Changing Climate, 2009. Homepage: http://www.ukcip-arcc.org.uk/

[17]P. D. Jones et al., UK Climate Projections science report: Projections of future daily climate for the UK from the Weather Generator, 2009. Homepage: http://ukclimateprojections.defra.gov.uk

[18]A. Peacock, D. Jenkins, and D. Kane, Investigating the potential of overheating in UK dwellings as a consequence of extant climate change. Energy Policy, 38 (7), 2010, pp. 3277 - 3288.

[19] http://www.metoffice.gov.uk/weather/uk/heathealth/

[20]P. J. Robinson, On the Definition of a Heat Wave, Journal of Applied Meteorology (American Meteorological Society) 40 (4), April 2001, pp. 762-775.

[21]M. Perry, A spatial analysis of trends in the UK climate since 1914 using gridded datasets. Climate Memorandum No. 21, Met Office, Exeter, 2006, pp. 29. 